\title{
Finite-Element Modeling of Intrastromal Ring Segment Implantation into a Hyperelastic Cornea
}

\author{
Sabine Kling and Susana Marcos
}

Purpose. Intrastromal corneal-ring segments (ICRSs) are applied to improve highly distorted vision in keratoconic, myopic, and astigmatic patients. Selections of ICRS geometry and position are primarily based on empirical nomograms. We developed a finite-element model (FEM) predicting the corneal response to different ICRS geometries in normal and keratoconic corneas.

Methods. A two-dimensional FEM was built in proprietary software (ANSYS-APDL), consisting of hyperelastic ocular tissues (cornea, limbus, sclera) and a triangular/hexagonal ICRS of poly(methyl methacrylate). An incrustation model was developed considering the local material addition and rigidity increase at the ICRS position and also for the triangular ICRS the geometric difference between its base (plane) and corneal tunnel (parallel to corneal surface). Different ICRS heights (150-350 $\mu \mathrm{m})$ and optical zones $(4.4-6.6 \mathrm{~mm})$ were simulated. An axis-symmetric model of keratoconus was studied, where corneal elasticity was decreased locally.

Results. ICRS geometry (height and optical zone) had a significant influence on corneal power: changes from 4.08 to -17.7 diopters (D) (healthy)/3.31 to $-20.5 \mathrm{D}$ (keratoconic) were observed. Central corneal thickness was predicted to increase by up to $38.5 \mu \mathrm{m}$ (healthy)/97.9 $\mu \mathrm{m}$ (keratoconic). Spherical aberration also changed upon ICRS implantation. The protrusion of the posterior cornea behind the rings was well predicted. The model confirmed the clinically reported trends on the effect of ring geometry.

Conclusions. FEM is a powerful tool to study the corneal response to ICRS implantation. The combination of FEM with individual biomechanical properties and geometry of patients holds promise to increase the predictability of ICRS surgery. (Invest Ophthalmol Vis Sci. 2013;54:881-889) DOI:10.1167/ iovs.12-10852 ntrastromal corneal-ring segments (ICRSs) are currently used Ias a refractive technique to correct moderate to high myopia $^{1,2}$ and as a therapeutic tool to improve the regularity of the aberrated corneal shape in astigmatic and keratoconic eyes. ${ }^{3}$ In the latter, ICRSs also stabilize the weakened cornea. The first implants of peripheral intracorneal rings in humans Spain.

From the Instituto de Óptica "Daza de Valdés" (CSIC), Madrid,

Supported by Spanish Government Grants FIS2008-02065, FIS2011-25637, and CENIT CEN-20091021, and European Research Council Grants ERC-2011 AdG-294099 (SM) and FPI-BES2009-024560 (SK).

Submitted for publication August 27, 2012; revised December 21, 2012; accepted December 21, 2012.

Disclosure: S. Kling, None; S. Marcos, None

Corresponding author: Sabine Kling, Instituto de Óptica "Daza de Valdés" (CSIC), Calle de Serrano 121, 28006 Madrid, Spain; kling.sabine@gmail.com. were reported in $1966 .{ }^{4}$ Later advances included changes in ICRS geometry (different arc lengths and heights, adjustable rings $^{5,6}$ ) and materials (synthetic gels ${ }^{6,7}$ or rigid materials ${ }^{8}$ ). Today, the most frequently implanted ICRSs are made of poly(methyl methacrylate) (PMMA), have a triangular ("Keraring"; Mediophacos Luda, and "Ferrara"; Ferrara Ophthalmics, both Belo Horizonte, Brazil) or hexagonal ("Intacs"; Addition Technology, Des Plaines, IL) shape and are available in several dimensions. For implantation, a tunnel parallel to the cornea is created at $70 \%-80 \%$ corneal depth, ${ }^{9}$ in which the ICRS is subsequently inserted.

Clinical studies report curvature changes of up to -13.75 diopters (D) after ICRS implantation, ${ }^{10}$ as well as a small rotation of the ICRS. ${ }^{2,11}$ Clinically, the ICRS height and optical zone are modified to modulate the desired refractive change, typically from empirical nomograms. To our knowledge only a few analytical ${ }^{5,12-14}$ and one numerical study (Pinsky PM, et al. IOVS 1995;36:ARVO Abstract S308) have been proposed to model the response of the cornea to the ICRS. The studies suggest a linear relation between refractive change and ICRS height (the higher the ICRS, the more effective) and optical zone (ICRS inner distance to the apex, the smaller the more effective). This relation is also reflected in the clinically applied nomograms. ${ }^{15}$ Although the biomechanical mechanisms underlying ICRS implantation have been approached, modeling relies strongly on the mechanical properties assigned to the corneal tissue and assumptions within the model. Corneal biomechanical models have improved in sophistication, from a single-membrane model with linear elastic ${ }^{16}$ or viscoelastic ${ }^{17}$ material properties, to hyperelastic orthotropic shell models that account for the tissue's microstructure, ${ }^{18}$ as well as solid viscoelastic (Kling S., EVER, 2011, Instituto de Óptica, CSIC) and nonlinear ${ }^{19}$ corneal models. Recently, the finite-element model (FEM) has also been suggested to model keratoconic corneas. ${ }^{20}$ The microstructure of corneal tissue is dominated by collagen fibers (mainly type $\mathrm{I}^{21}$ ) that provide the necessary stability to maintain the spherical shape of the cornea. Keratoconus is generally thought to be associated with a local loss of collagen fibril orientation. ${ }^{22,23}$ This results in a local decrease of corneal rigidity ${ }^{24,25}$ and leads to the deformation in form of a cone. ${ }^{26}$ The cone is typically slightly displaced interiorly and temporally from the corneal apex, ${ }^{24}$ resulting in increased corneal aberrations ${ }^{26,27}$ and a highly distorted vision. ${ }^{28}$ Keratoconic corneas with central cones were reported to occur in approximately 29 of the patients.

Recently, new quantitative imaging techniques have allowed analyzing geometric changes of the cornea before and after ICRS implantation. ${ }^{11}$ Advanced imaging also allows a better understanding of the biomechanical mechanisms occurring with ICRS implantation, as a better verification of the simulated results is possible. We have developed a solid hyperelastic FEM of the anterior eye segment and studied the effect of ICRS implantation as a function of ICRS geometry and implantation parameters, for both normal corneas and a simple 


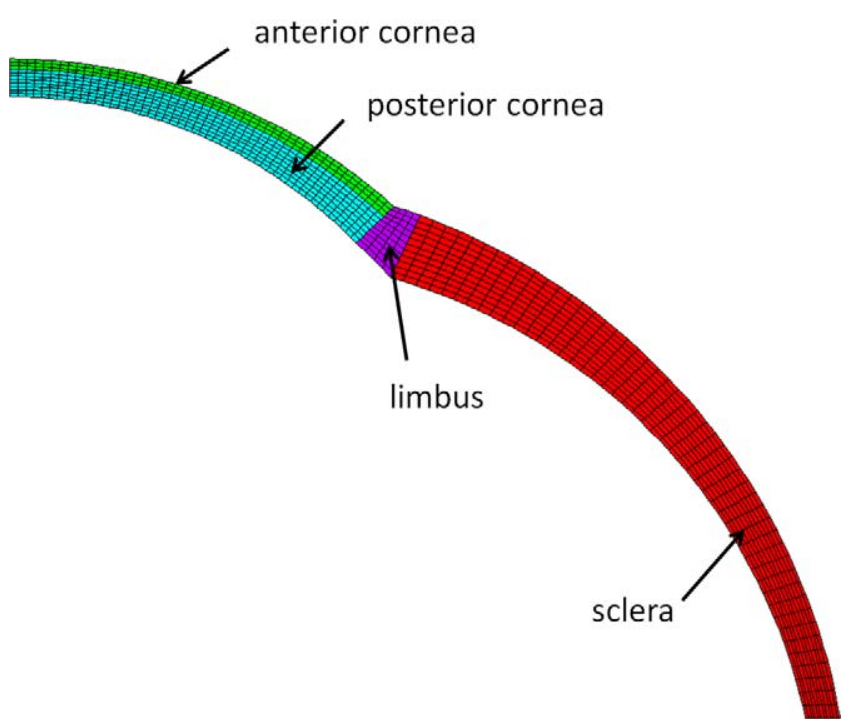

Figure 1. FEM geometry and mesh, consisting of anterior and posterior cornea, limbus, sclera.

keratoconic model, with an altered elasticity profile. The model will reveal which parameters are most important to achieve the attempted refractive corrections after ICRS surgery. This will give a better understanding of the biomechanical response of the cornea and, hence, a more accurate prediction of the postsurgical results.

\section{METHODS}

\section{FEM-Cornea}

A two-dimensional axis-symmetric model was built with a hyperelastic material for the ocular tissues. The geometry was defined by the cross-section of the anterior quarter of the outer eye coat, consisting of cornea, limbus, and sclera (see Fig. 1). As previously found from experiments, ${ }^{30}$ the anterior cornea was set slightly stiffer (factor 1.1) than the posterior cornea. Preoperative corneal curvatures (anterior, posterior) and thickness $(538 \mu \mathrm{m})$ were adjusted to represent an emmetropic eye with homogeneous corneal refraction of $42.2 \mathrm{D}$ over a $6-\mathrm{mm}$ pupil. The calculations were also performed for a simplified keratoconic eye with a centrally lower corneal elasticity. We applied a three-parameter Mooney Rivlin hyperelastic material model, similar to that previously proposed by Pandolfi et al., ${ }^{18}$ with the following parameters: byp $1=1.500 \times 10^{3}$, byp $2=1.415 \times 10^{5}$, byp $3=2.139 \times 10^{7}, d=0$.

The cornea was considered almost noncompressible ${ }^{31}$ and thus Poisson's ratio was set to 0.5 . Corneal density ${ }^{32}$ was set to $1062 \mathrm{~kg}$ / $\mathrm{m}^{3}$. The sclera was modeled with a uniform Young's modulus of 33.6 $\mathrm{MPa}$, set within the previously reported range (17-60 MPa). ${ }^{33}$ The thickness variations along the sclera (from the limbus to the equator) were taken from the literature for pig eyes. ${ }^{34}$ The scleral diameter was adjusted to human sclera $(23 \mathrm{~mm})$ and the limbus geometry was adjusted to match cornea and sclera, with material properties laying between both. A $15 \mathrm{~mm} \mathrm{Hg}$ pressure was applied to the posterior surfaces of cornea, limbus, and sclera, simulating physiologic intraocular pressure (IOP). Constraints were set at the scleral equator, permitting axial expansion to represent the natural eye fixation by the ocular muscles. A quadrilateral mesh was created for all geometric parts, resulting in approximately 1122 elements: 550 for cornea, 44 for limbus, and 528 for sclera (see Fig. 1). The actual number of elements was dependent on the small variations in ICRS height, width, and corneal tunnel dimensions.

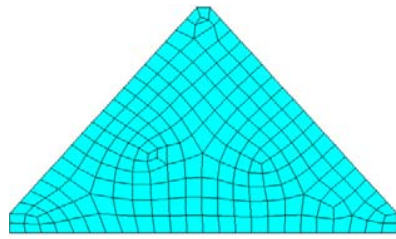

triangular ICRS

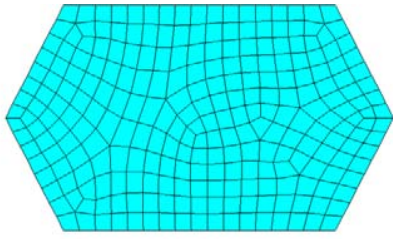

hexagonal ICRS
FIGURE 2. ICRS geometry and mesh of triangular and hexagonal crosssections (height: $350 \mu \mathrm{m}$ ).

\section{FEM Modeling of ICRSs}

ICRSs of triangular and hexagonal sections were modeled. Different ICRS heights and optical zones (defined as the distance from the inner ICRS edge to the corneal apex) were simulated, consistent with dimensions available on the market, including: 4.4, 5.0, 5.4, 6.0, and $6.6 \mathrm{~mm}$ optical zones; $150-350 \mu \mathrm{m}$ heights $(\Delta=50 \mu \mathrm{m})$, and $600 \mu \mathrm{m}$ width. The ICRS material was PMMA, an isotropic polymer with a Young modulus of $1800 \mathrm{MPa}$, a Poisson's ratio of 0.48 , and a density of $1170 \mathrm{~kg} / \mathrm{m}^{3}$.

Triangular Cross-Sectional ICRSs. For the triangular ICRS the edges were slightly rounded, consistent with the actual shape of the ring, as reported from noncontact profilometric shapes of ICRS samples (Ferrara Rings and KeraRings; Pablo Pérez-Merino and Susana Marcos, personal communication, 2009). A quadrilateral mesh was created for the ICRS consisting of 90 to 204 elements, depending on ICRS height. Triangular ICRSs are fabricated with a plane base; thus, the geometry was not further modified (see Fig. 2A).

Hexagonal Cross-Sectional ICRSs. For the hexagonal ICRS the edges were set at $1 / 6$ width. The quadrilateral mesh consisted of 121 to 252 elements. Hexagonal ICRSs are fabricated with a tilted plane, adapted to the corneal curvature. This was implemented by rotating the ICRS by $-22^{\circ}$ (with respect to the position shown in Fig. 2B) for implantation.

\section{Model Description}

For ICRS implantation three steps were considered: (1) Local material addition (ICRS), causing a local displacement of the corneal tissue. Friction between corneal tissue and ICRS was neglected. (2) Local stiffness increase due to PMMA insertion. (3) Applying only to triangular cross-sectional rings, disagreement between the orientation of the ICRS base with respect to the corneal tunnel, since the corneal tunnel is cut parallel to the corneal surface, but the ICRS is manufactured with a plane base

\section{Simulation}

The simulation of the ICRS was performed following an incrustation method, using parameters similar to those used for clinical implantation. In a first step the tunnel was created at $75 \%$ depth of the cornea (oriented parallel to the corneal curvature) like that in surgery $(70 \%-80 \%)$. Clinically, this is done either manually (separating collagen layers with a mechanical device) or with the use of a femtosecond laser. We simulated the tunnel by a theoretical gap between the upper and lower adjacent corneal areas, with a width slightly larger than that of the ICRS width. Although clinically the tunnel width is usually cut with a width similar to that of the ICRS, the corneal tunnel can be extended centrally toward the central visual axis or eccentrically toward the limbus (especially with manual tunnel creation). ${ }^{35} \mathrm{~A}$ wider tunnel was also consistent with a more convergent simulation. The inner tunnel distance to the apex was set according to the optical zone of the ICRS. In a next step the ICRS geometry was placed within the cornea: for triangular ICRSs with the center of its base at the center of the tunnel; for hexagonal 

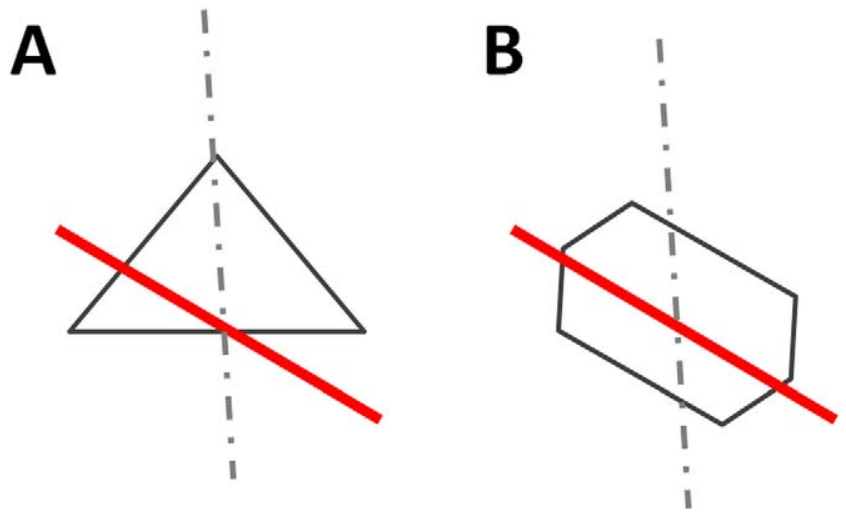

Figure 3. Positioning of the ICRS with respect to the tunnel (in red) within the cornea. (A) The triangular ICRS is positioned with the center of its base at the center of the tunnel. (B) The hexagonal ICRS is positioned with its center at the center of the tunnel.

ICRSs with its center at the center of the tunnel (see Fig. 3). Contacts without friction between the corneal tissue and the ICRS were simulated by flexible-flexible contact pairs $^{36}$ with a pinball region (contact search size) of 100 times the size of one finite element. Ramped effects of collision (i.e., linear increase of acting forces when establishing contacts) were defined between the ICRS surfaces and the tunnel surfaces. The response was analyzed after the ICRS had moved as an effect of establishing the contacts. Because viscoelastic material properties were not considered, the predicted deformed corneal shape describes the long-term effect of ICRS implantation.

\section{Keratoconic Model}

We studied a simplified keratoconic model consisting of an axissymmetric cone. It is known from previous studies that keratoconic corneas exhibit a disorder of collagen fiber orientation at the location of the cone likely resulting in a local reduction of corneal tissue rigidity $^{22,23}$ and resulting in a large local deformation. A hyperelastic material considered the nonlinear material properties of the corneal tissue with strain. In this study we investigated how local stiffness variations across the cornea affect the result of ICRS implantation. We simulated a pathologic cornea consisting of a central weakened zone, of different simulated diameters. Under the application of normal IOP $(15 \mathrm{~mm} \mathrm{Hg})$ this led to the typical formation of a cone. Similar simple FEM models to mimic the local reduced elasticity modulus in keratoconus and its treatment by cross-linking have been recently presented by Roy and Dupps. ${ }^{20}$ Corneal weakening was implemented by reducing the three hyperelastic parameters (byp 1-3) by a factor of $F$ $=100$ in an angular sector of the cornea (defined by the angle from the apex, at $90^{\circ}$, toward the periphery, with the origin at the center of curvature of the cornea). Different cone extents were studied. Unless otherwise noted, the angular extent of the cone in keratoconic corneas was $7^{\circ}$ (corresponding to a cone radius in the anterior cornea of 1.00 $\mathrm{mm}$ ). The amount of weakening was determined by reducing $F$ until the IOP produced a deformation leading to a corneal power of $52.6 \mathrm{D}$ at the cone; this refraction corresponds to a stage II keratoconus in the Amsler-Krumeich scale. The deformation was consistent with a decrease in corneal thickness by $58.7 \mu \mathrm{m}$. Note, the tunnel was created before applying the IOP; thus, it will deform asymmetrically depending on the dimensions of the weakened tissue.

\section{Analysis}

The effect of ICRS implantation was studied as a function of ICRS geometry, ICRS position, and changes in local corneal elasticity. The
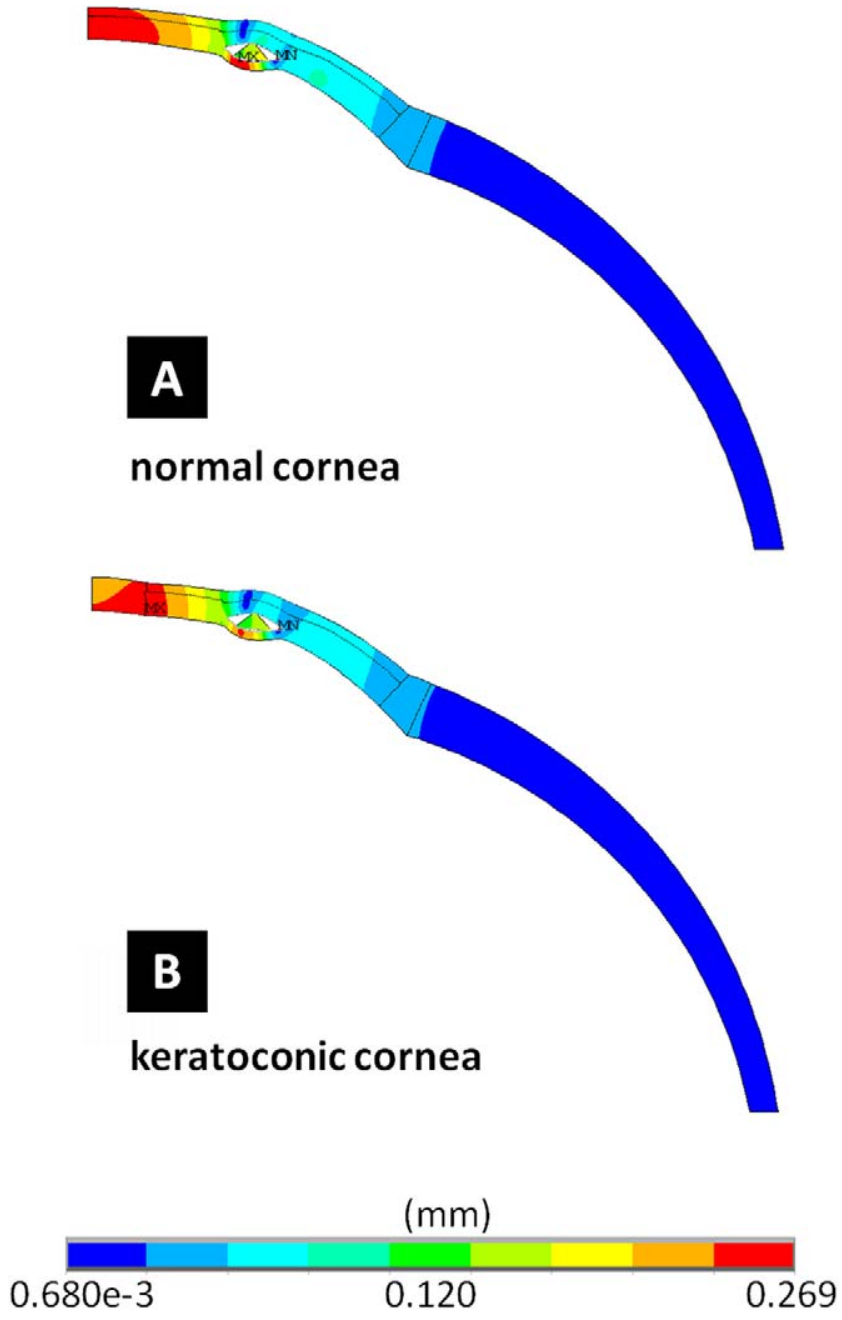

FIgURE 4. Absolute displacement after standard triangular ICRS (height: $300 \mu \mathrm{m}$; width: $600 \mu \mathrm{m}$; optical zone: $2.5-\mathrm{mm}$ radius) implantation in a normal (A) and in a keratoconic cornea (B).

outcome of the FE model was the deformed shape of the postoperative cornea (coordinates of the anterior and posterior surfaces).

Calculation of the Change in Refraction. Two different methods were used to estimate the change in corneal refraction: (1) changes in the anterior corneal surface only (such as those measured by keratometry), and (2) changes in the refraction resulting from both the anterior and posterior corneal surface (such as those measured by refracting the eye). For (1) the mean radius of corneal curvature in a central region of $4-\mathrm{mm}$ diameter was estimated by fitting a circle to the coordinates of the anterior surface (obtained from the FE model). The keratometer equations were applied to determine the mean corneal refraction, assuming a refractive index of $n=1.3375$ (as done in many commercial keratometers). Calculations were performed using a commercial program (Matlab; The MathWorks, Natick, MA). For (2), the change in corneal power (pre- and postsimulated implantation) was computed by ray tracing through the anterior and posterior corneal surface (coordinates obtained from the FEM analysis and fitted using extended cubic splines). The distance between both surfaces was set by the corneal thickness at the apex. Refractive indices of $n_{\text {Cornea }}=$ $1.376, n_{\text {Water }}=1.336$, and $n_{\text {Vacuum }}=1.000$ were assumed for the cornea, aqueous humor, and air, respectively. Calculations were performed using a commercial optical and illumination design software (Zemax 12; Radiant Zemax Corporate, Redmond, WA). Corneal power was estimated for different pupil diameters, ranging from 3- to 6-mm diameters. These calculations included both the contributions of 

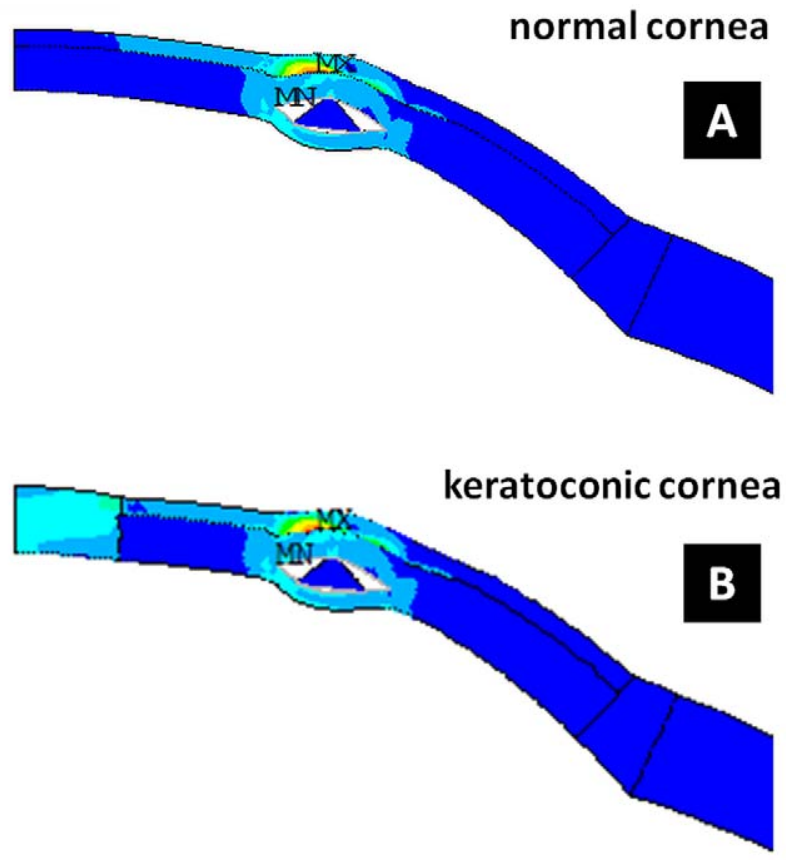

\section{$5040-4$}
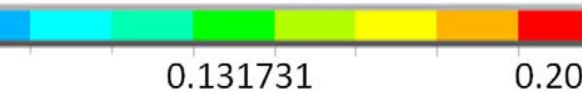

$0.504 \mathrm{e}-4$

0.131731

0.206331

Figure 5. First principal strain distribution after standard triangular ICRS (height: $300 \mu \mathrm{m}$; width: $600 \mu \mathrm{m}$; optical zone: 2.5 -mm radius) implantation in a normal (A) and in a keratoconic cornea (B).

corneal shape and potential changes in the displacement of the corneal apex. Changes in refraction were calculated considering that the retina does not move (i.e., an axial shift of the apex reduces the focal length of the eye).

Calculation of the Change of Spherical Aberration. Changes in the fourth-order Zernike spherical aberration were calculated using ray tracing. Aberrations were evaluated in the pupil plane at a distance of 3 $\mathrm{mm}$ from the cornea. The spherical aberration of the anterior corneal surface was estimated assuming a single surface (defined by the coordinates of the simulated cornea, fitted by cubic splines) and the corneal index of refraction $(n=1.376)$. The spherical aberration of the entire cornea was estimated on a two-surface model (implemented in Zemax as described above).

\section{Software}

Proprietary software (ANSYS APDL 14.0; ANSYS, Inc., Canonsburg, PA) was used for FEM simulations, a commercial program (MatLab R2010a, The MathWorks) was used to analyze the geometry of the initial and deformed cornea, and a commercial illumination design software (Zemax; Radiant Zemax Corporate) was used to determine the refractive changes and spherical aberration.

\section{Results}

FEM predictions for the deformed corneal shape were analyzed for normal and keratoconic corneas. The absolute relative displacement of the surfaces and the first principal strain distribution resulting from the simulated implantation of a standard ICRS of triangular section (height: $250 \mu \mathrm{m}$; width: 600 $\mu \mathrm{m}$; optical zone: $2.5 \mathrm{~mm}$ ) are shown in Figures 4 and 5, respectively.

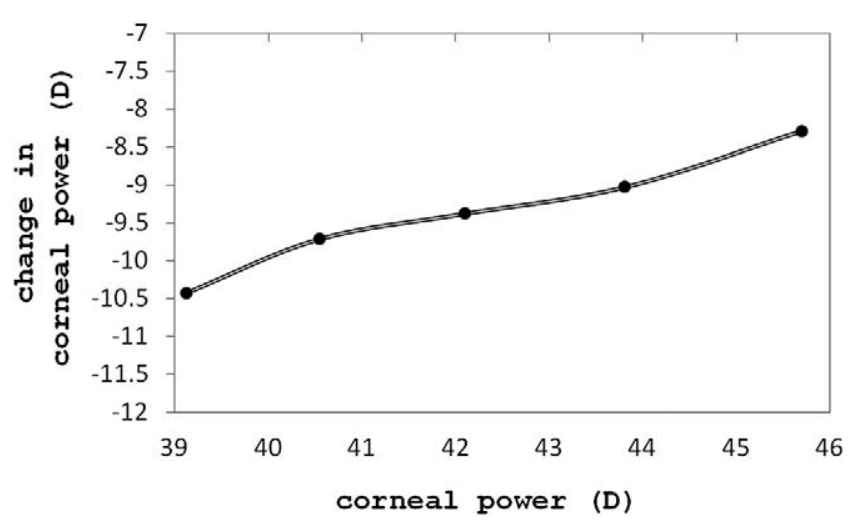

FIGURE 6. Changes in corneal power after standard triangular ICRS (height: $300 \mu \mathrm{m}$; width: $600 \mu \mathrm{m}$; optical zone: $2.5-\mathrm{mm}$ radius) implantation in a normal cornea as a function of the pre-op corneal power, analyzed for a pupil of 4-mm diameter.

The anterior cornea showed a smooth transition near the location of the ICRS position, compared with the marked protrusion of the posterior cornea behind the ICRS. Although the geometric changes of the cornea were highly impacted by the geometry of the ICRS (see next subsections), preoperative corneal geometric parameters (such as the anterior corneal curvature) also played a role in the refractive changes after surgery. Figure 6 shows the effect of preoperative corneal curvature on refractive correction, with the higher corrections achieved with flatter corneas $(-0.326 \mathrm{D}$ less refractive correction per $1 \mathrm{D}$ increase in curvature).

\section{Changes in Corneal Thickness}

Corneal thickness at the apex increased after ICRS implantation between 3.91 and $38.5 \mu \mathrm{m}$ for the normal cornea and between 66.8 and $97.9 \mu \mathrm{m}$ for the keratoconic cornea, depending on ICRS geometry (see Fig. 7). The higher ICRSs produced the largest increase in corneal thickness. Also hexagonal ICRS cross-sections increased central thickness less than that of triangular cross-sections.

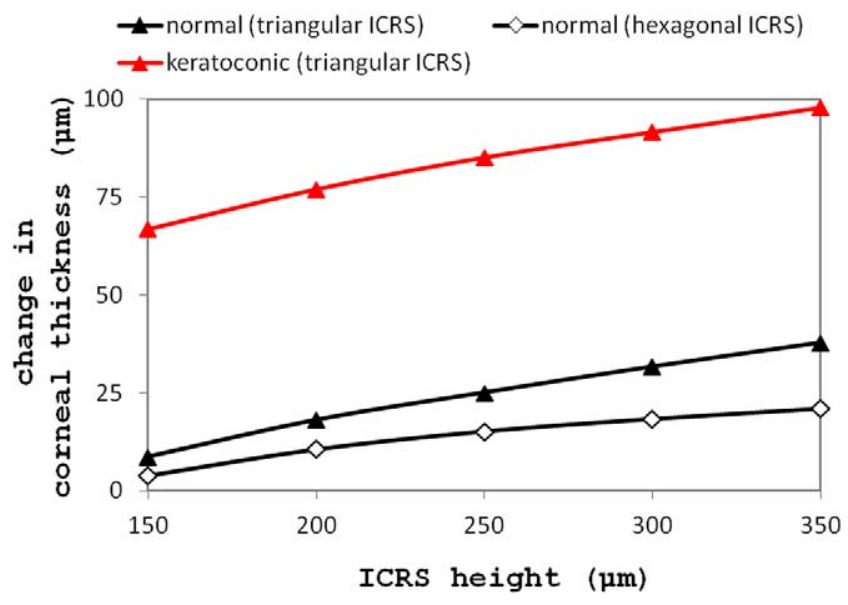

FIgURE 7. Changes in corneal apical thickness after ICRS (width $=600$ $\mu \mathrm{m})$ implantation in keratoconic and normal corneas, as a function of ICRS height (150-350 $\mu \mathrm{m})$; independent of optical zone dimensions. The simulation in the normal cornea is for triangular and hexagonal ICRSs, and for triangular ICRSs in the keratoconic cornea is for a triangular ICRS. 

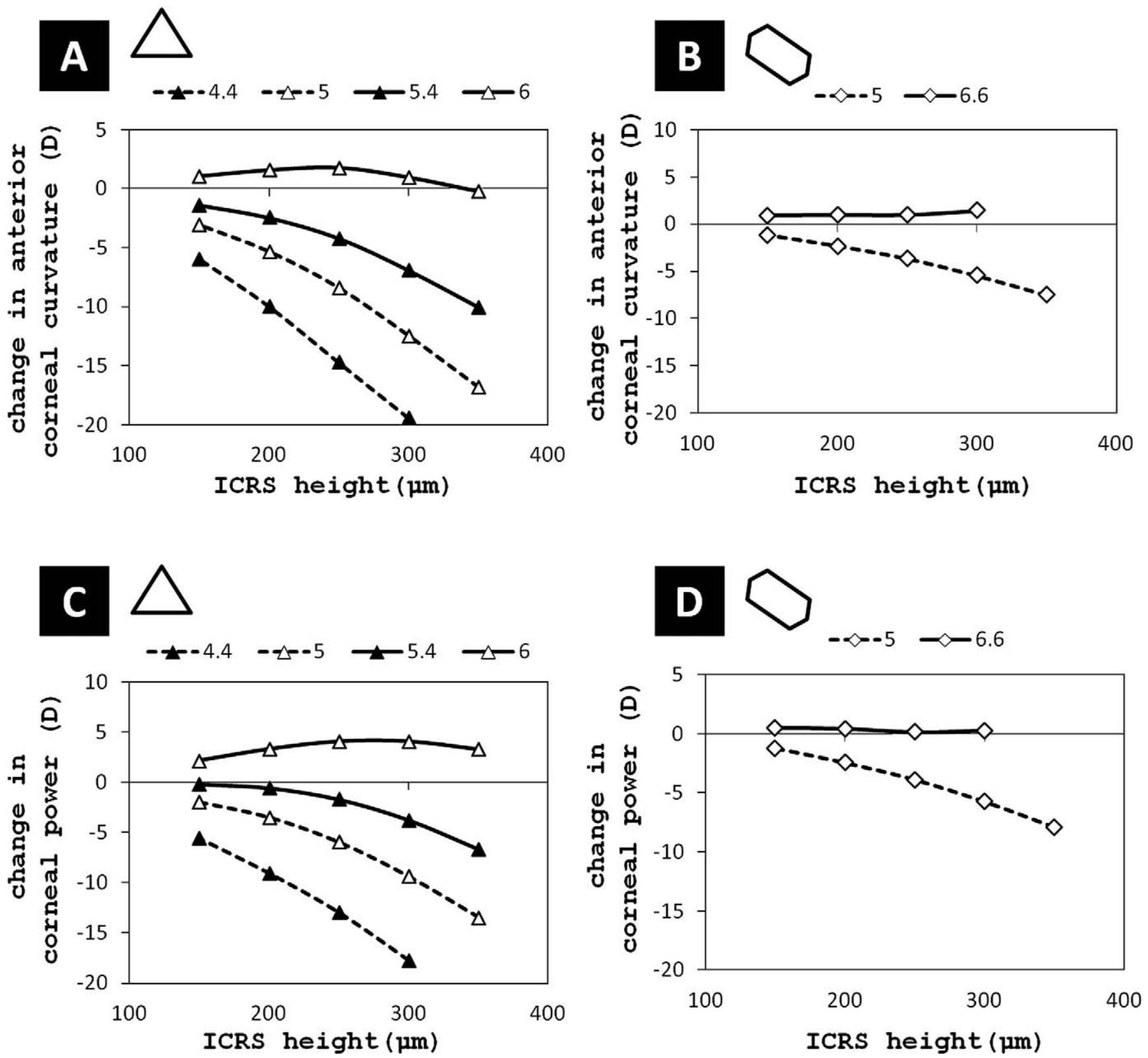

Figure 8. Normal cornea: changes in anterior corneal curvature $(\mathbf{A}, \mathbf{B})$ and changes in corneal power $(\mathbf{C}$, D) after ICRS $($ width $=600 \mu \mathrm{m})$ implantation as a function of ICRS height for different optical zone diameters (4.4-6.6 mm) and cross-sections ([A, C]: triangular; [B, D]: bexagonal). Data are for 4-mm-diameter pupil.

\section{Effect of ICRS Geometry on Anterior Corneal Curvature}

Normal Corneas. Figure 8 shows the effect of the ICRS on the change on the anterior corneal curvature (top row, Figs. $8 \mathrm{~A}, 8 \mathrm{~B}$ ) and the total corneal power (bottom row, Figs. 8C, $8 \mathrm{D})$, as a function of ICRS height, for different optical zone diameters (4.4-6.6 mm). Simulations were performed for triangular ICRS (left panels, Figs. 8A, 8C) and hexagonal ICRSs (right panels, Figs. 8B, 8D). Data are for a pupil of $4-\mathrm{mm}$ diameter. The largest changes in anterior corneal curvature occurred for smaller optical zones (for a fixed ICRS height). ICRS with the largest optical zones (6 $\mathrm{mm}$ or larger) appeared to be ineffective in producing a change in curvature. Also, changes in anterior corneal curvature increased with ICRS height (for a fixed optical zone). Corneal power changed in a manner similar to that of the anterior corneal curvature, with slightly less negative refractive change, consistent with the positive contribution of the posterior surface. Hexagonal ICRSs produce lower changes than those produced by ICRSs with a triangular cross-section, but they also show an increase with ICRS height and a decrease with optical zone diameter.

Keratoconic Corneas. Figure 9 shows the effect of a triangular ICRS on corneal power on a keratoconic cornea. The curvature changes as a function of optical zone are similar to those found in normal corneas, whereas curvature changes as a function of ICRS height are slightly larger than those in normal corneas. Hexagonal ICRSs were not analyzed for keratoconic corneas, in that the triangular section ICRSs are more often used in clinics in the treatment of keratoconus. For a typical ICRS geometry used clinically (triangular section, $250 \mu \mathrm{m}$ height, 5-mm-diameter optical zone) our simulations predict a corneal power change close to $-10 \mathrm{D}$. 


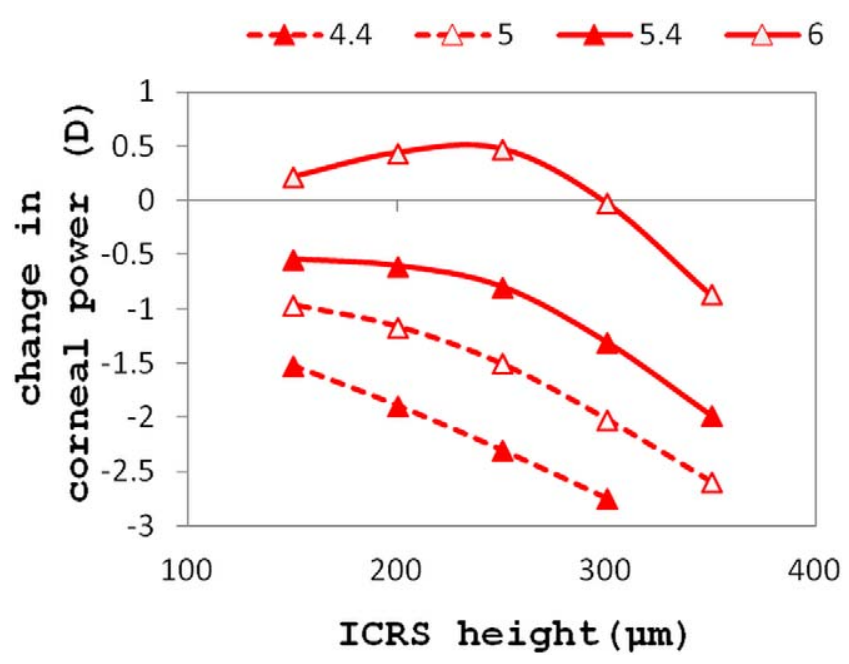

FIGURE 9. Keratoconic cornea: changes in corneal power after ICRS implantation (width $=600 \mu \mathrm{m}$ ) as a function of ICRS height for different optical zone diameters $(4.4-6.6 \mathrm{~mm})$. Data are for a 4-mmdiameter pupil.

\section{Refractive Changes: Contributions to Their Change}

The changes in refraction are due to changes in curvature of both the anterior and posterior corneal surfaces, as well as changes in the relative position of the corneal apex. Figure 10 shows the relative contributions of the anterior and posterior corneal surfaces, and the relative shift of the apex (which ranges from $0.637 \mathrm{~mm}$ for the smallest optical zone and thinnest ICRS to $1.08 \mathrm{~mm}$ for the largest optical zone and highest ICRS) to the refractive change, after implanting a triangular 5-mm optical zone ICRS in a normal cornea. The anterior surface and the apex reduced corneal refraction, whereas the deformations of the posterior surface increased corneal refraction. On average, changes in the anterior cornea, posterior cornea, and apex shift contributed by $-75.4 \%$, $+12.3 \%$, and $-13.4 \%$, respectively, in a normal cornea.

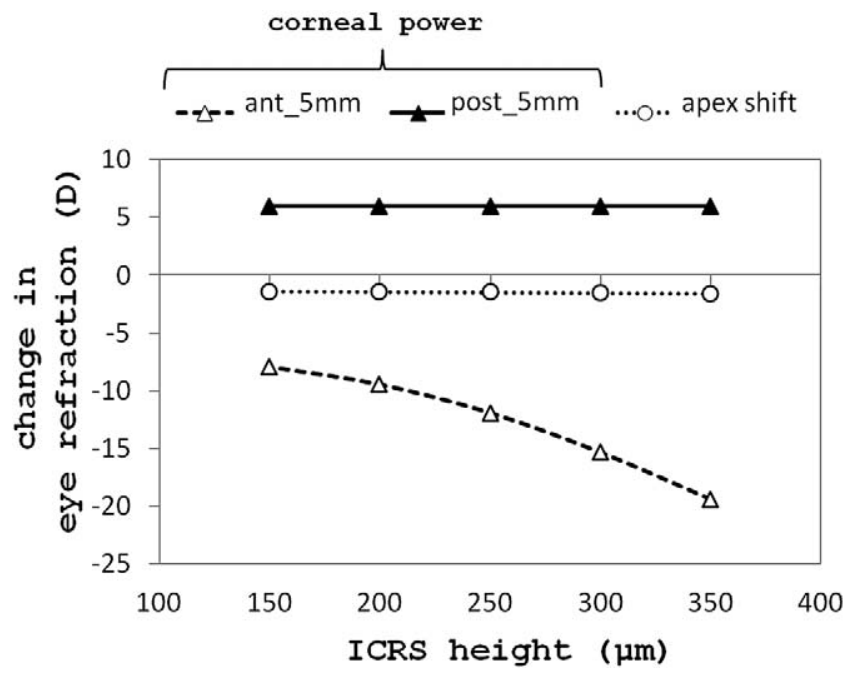

Figure 10. Contribution of the anterior and posterior corneal curvature and of the corneal apex shift to the absolute refractive change, after implantation of a triangular ICRS (width: $600 \mu \mathrm{m}$; 5-mm optical zone diameter) in a normal cornea. Data are for a 4-mmdiameter pupil.

\section{Changes in Spherical Aberration}

Normal Corneas. Figure 11A shows the change of the corneal spherical aberration (SA) for different optical zones, for a 4-mm-diameter pupil. SA was negative (consistent with a corneal prolate shape), its absolute value increased with ICRS height (between -0.5 and $-1.5 \mu \mathrm{m}$ ), and was stable across optical zones (in all cases larger than the pupil diameter under test).

Keratoconic Corneas. ICRSs implanted in a simulated keratoconic cornea also induced SA, with higher amounts in most cases than those in normal corneas (up to $-3.5 \mu \mathrm{m}$ ). Unlike in normal corneas, the largest absolute amount of SA was not induced with the highest ICRSs, but ICRSs with 200$250 \mu \mathrm{m}$ height (see Fig. 11B). Also the induced SA varied with optical zone, with the larger optical zone inducing the largest amount of SA.

\section{Effect of Keratoconus Size (Axis-Symmetric Model)}

The effect of ICRS implantation on corneal power was highly dependent on cone size. Figure 12 shows the effect of a standard triangular ICRS on the corneal reshaping of keratoconic corneas with weakened central area of different angular extents. The cornea was effectively flattened for cone extents smaller than $13^{\circ}$, but actually steepened for larger cones, with the corneal power change following a sinusoidal pattern as a function of cone size. The first point in the graph $\left(0^{\circ}\right)$ corresponds to a normal cornea. The induced spherical aberration also changed with cone size, reaching a maximum (absolute value) for a cone size of $17.5^{\circ}$.

\section{Discussion}

We present a finite-element model that identifies the most important parameters determining corneal deformation (and hence the change in refractive power) after ICRS implantation. Most previous predictions of ICRS-induced refractive changes were based on analytical, ${ }^{5,12-14}$ numerical (Pinsky PM, et al. IOVS 1995;36:ARVO Abstract S308), or empirical regression models. ${ }^{15}$ Fleming et al. ${ }^{5}$ considered only the anterior corneal shape for estimations of the corneal refractive change. Although we can confirm that this is on average the largest factor $(75.4 \%)$, our calculations also show that the posterior surface (11.3\%) and the corneal apex shift (13.4\%) contribute significantly to the finally achieved refractive change after ICRS implantation.

Although our model predicted the overall refractive outcomes found in patients, we observe slightly larger differences in the range of predicted refractive corrections (from 4.09 to $-17.71 \mathrm{D}$ in our model, for a $4-\mathrm{mm}$ pupil) in comparison with clinical results $\left(-1.0\right.$ to -6.0 D). ${ }^{1,2}$ Differences may arise from the assumed input biomechanical properties of cornea, limbus, and sclera, as well as from the geometric approximations in the model. We performed axis-symmetric simulation assuming an ideal spherical outer eye coat and a $360^{\circ}$ ICRS. Assuming an ideal spherical eye coat can be justified for myopic corrections in normal eyes. Also, an axis-symmetric model for keratoconus is representative of a centrally located keratoconus, which accounts for approximately $25 \%$ of the keratoconic population. ${ }^{29}$ Although a larger part of keratoconic corneas are asymmetric, the predictions of our symmetric model are consistent with clinical observations found in keratoconic patients implanted with ICRS: (1) ICRSs are more effective for smaller optical zones, (2) and there is an almost linear relationship between ICRS height and the amount 
$\mathbf{A}$

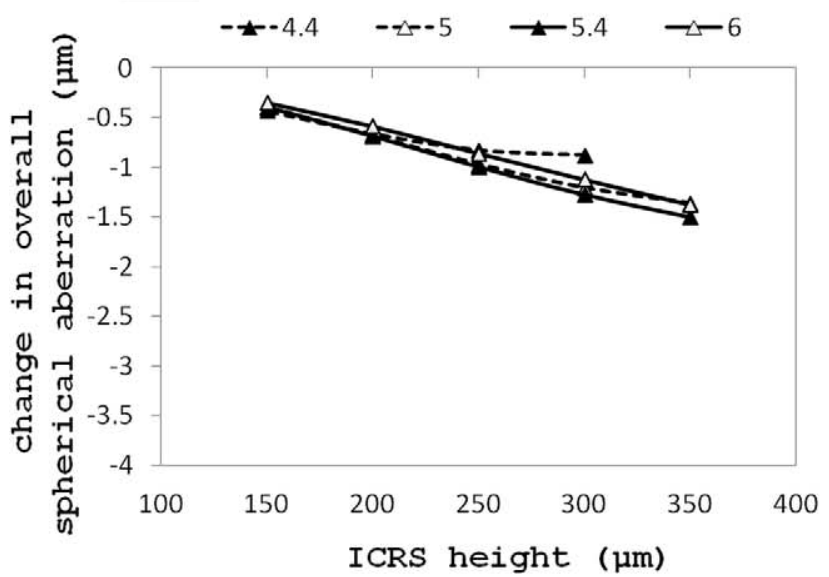

B

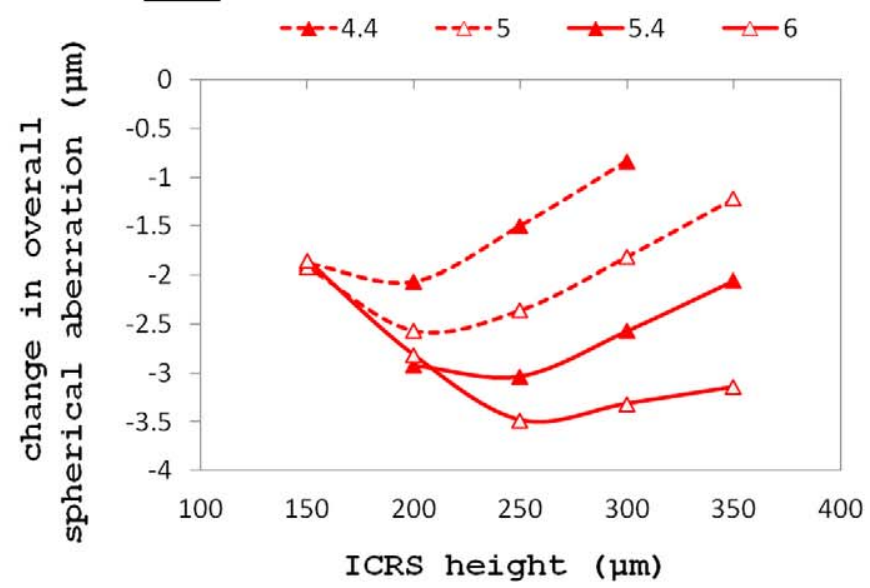

FIGURE 11. Changes of spherical aberration in a (A) normal and (B) keratoconic cornea after implanting triangular ICRSs (width $=600 \mu \mathrm{m})$ as a function of ICRS height for different optical zone diameters (4.4-6 mm). Data are for 4-mm-diameter pupil.

of refractive decrease after implantation. We also found the triangular ICRS to be more effective than the hexagonal ICRS, which confirms previous clinical observations. ${ }^{9}$ We found changes in corneal power of keratoconic corneas of 3.31 to $-20.5 \mathrm{D}$, which are consistent with keratometric reports from the literature $(4.56$ to $-13.75 \mathrm{D}) .{ }^{10}$ Also the predicted changes in spherical aberration $(-0.34$ to $-3.07 \mu \mathrm{m})$ from our simulations fall in the range of what is reported clinically $(-0.17$ to $-0.40 \mu \mathrm{m}){ }^{37}$ We observed an axial shift of the corneal apex after ICRS implantation (up to $-1.08 \mathrm{~mm}$ ). This effect has also been observed clinically, reporting a decrease in the anterior chamber depth after ICRS implantation. ${ }^{38}$ As we could confirm in our simulations, the conical deformation in keratoconus makes the eye longer, ${ }^{39}$ and the overall axial length of the eye decreases upon ICRS implantation. Our model also predicts the trend toward increased corneal thickness after ICRS implantation ${ }^{11}$ as a result of material displacement toward the corneal apex, by 3.91 to $97.9 \mu \mathrm{m}$ (depending on the ICRS geometry and optical zone), which falls in the range of clinical observations $\left(17 \mu \mathrm{m},{ }^{40} 76 \mu \mathrm{m}^{11}\right)$. Due to the geometric shape of the ICRS, the central corneal

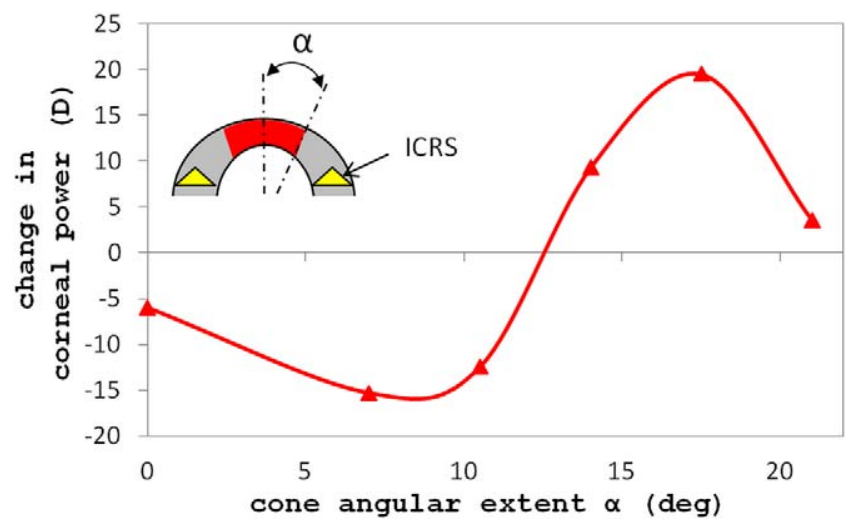

FIGURE 12. Changes in corneal power after standard triangular ICRS (height: $300 \mu \mathrm{m}$; width: $600 \mu \mathrm{m}$; optical zone: $5-\mathrm{mm}$ radius) implantation in a cornea with a central weakened zone of different angular extents $(\alpha): 0^{\circ}$ (normal cornea) to $20^{\circ}$. The insets illustrate the definition of the angular dimensions of the cone. thickness increase was more evident with triangular than with hexagonal cross-section (see Fig. 7).

In previous studies it was speculated that the ICRS rotates within the cornea, because its base is observed to be oriented with a certain angle respect to the corneal surface. ${ }^{2,11}$ Also an accumulation of cells at the ICRS edges has been observed using microscopy, ${ }^{41}$ and tissue wound healing was attributed to induce the rotation. However, the disagreement between the orientation of ICRS base and corneal tunnel (triangular ICRSs only) is not a true rotation but rather a result from the plane shape of the rigid ICRS being inserted into the curved soft cornea. Additional smallest rotations moving the ICRS base away from its horizontal orientation may occur due to acting forces along the ICRS or the mentioned healing process. This effect is much smaller than the disagreement in orientation between the ICRS base and the corneal tunnel $\left(5-12^{\circ 10}\right.$ vs. $45^{\circ}$ [angle between corneal surfaces and ICRS base]) and can also be observed in the simulations (see Figs. 4, 5).

The biomechanical changes occurring with keratoconus are still largely unknown, although a decrease in ocular rigidity in keratoconic eyes has been reported. ${ }^{25,39}$ This probably results in a local stretching that would explain the decreased corneal thickness at the cone. We studied the effect of decreasing the hyperelastic parameters, which result in a decrease in corneal stiffness and a local change in curvature. Previous studies ${ }^{20,42}$ simulated the weakened keratoconic cornea by decreasing stiffness and corneal thickness separately. However, ideally, the change in mechanical parameters would cause the out-bulging and thinning at once. We captured this aspect in our model by decreasing corneal stiffness until the IOP led to the formation of a cone. Note that this weakening refers to the unloaded cornea. As known from stress-strain graphs, the elastic modulus of hyperelastic materials depends on the amount that the material has been stretched. We observe highest strains at the position of the cone, which causes a local hardening of the corneal tissue compared to its unloaded condition. From estimations from our model, the Young's modulus of the stressed keratoconic cornea is approximately 10 times weaker than the normal cornea. Experimentally, the keratoconic cornea is estimated to be $0^{43}$ to $2.04^{44}$ weaker than normal cornea. The fact that this is the average across keratocones of different severity might justify the need of stronger weakening for a $52.6 \mathrm{D}$ cone like that in our simulation. Our axissymmetric keratoconic model described the geometric chang- 
es of a central keratoconus, and allowed us to analyze the effect of implanting an ICRS with an altered elasticity profile and the corresponding conical deformation. This is accurate for centrally located keratocones and a good approximation for asymmetric keratocones, because the cone is always centered within the ICRS. Very interestingly, apart from the geometric parameters of the ICRS and the optical zone, the extent of the cone played a primary role in the effectiveness of the ICRS. The lack of effectiveness (and actual sign reversal in the corneal power range) for larger cones may explain, at least in part, the high outcome variability found clinically. ${ }^{8-10,15}$

A limitation of our model is the lack of conformity of the corneal tissue to the ICRS shape, unlike the evidence from in vivo OCT images in corneas implanted with ICRSs implanted in corneas. ${ }^{11}$ Nevertheless, Twa and colleagues ${ }^{45}$ observed new collagen formation, increased keratocyte density, and lipid accumulation adjacent to the ICRS after implantation. We suggest that these processes might fill the free space observed in our simulation. Interestingly, the local shape of the anterior and posterior cornea in front and behind the ICRS observed in recently published fully quantitative corneal OCT images of corneas implanted with ICRSs ${ }^{11}$ is very well predicted by the model. Our model relied on the assumption of corneal mechanical parameters available from the literature. However, these parameters (in particular the Young's modulus) varies up to two orders of magnitude. ${ }^{19,46-49}$

The predictability of our simulations will benefit from an increased accurate knowledge of the mechanical parameters of individual patient corneas. Newly developed systems ${ }^{42,50,51,53,54}$ give hope that in vivo measurement of corneal elasticity will be possible soon: (1) optical coherence tomography (OCT) ${ }^{11,54}$ allows accurate measurements of corneal and limbus geometry, which are essential input parameters to FE models. Furthermore, OCT is useful to evaluate the outcomes of the model. (2) Brillouin microscopy has allowed in vivo estimates of the local elasticity map across the cornea. $^{50}$ (3) Second-harmonic microscopy (so far only applicable in vitro due to high-level light exposures) allows imaging the collagen fiber organization in the intact cornea. ${ }^{52,53}$ (4) Dynamic corneal imaging in response to an airpuff response analysis allows gaining insights into dynamic corneal biomechanical properties in vivo. ${ }^{51}$

Our model predicts the effects of ICRS geometry on the corneal biomechanical response, which will benefit from more refined models and customized corneal geometric and mechanical parameters. Besides, the model gives insights on some surgical aspects that could be modulated to improve the refractive outcomes. For example, the model predicts that the relative orientation of the ICRS within the cornea plays a role in the corneal reshaping. Also, preliminary simulations suggested an increased effect of ICRS, when the tunnel is cut with a diameter slightly smaller than the diameter of the ICRS. Finally, the 2D axis-symmetric model could be extended to 3D to allow modeling of very asymmetric corneas, frequent in keratoconus.

In summary, finite-element simulation of ICRS implantation allows identifying important parameters that determine corneal reshaping and refractive outcomes. This model permits a more detailed prediction of the surgical outcomes than currently used estimations, and thus a more systematic selection of the most appropriate ICRS parameters as well as a better understanding of the mechanisms involved in the biomechanical corneal response to surgery. This approach will become especially important as biomechanical models of the cornea become more sophisticated and incorporate corneal biomechanical properties of individual patients from emerging quantitative imaging systems.

\section{Acknowledgments}

The authors thank Nándor Békési and Alberto de Castro for scientific discussions and technical advice, and are thankful for the use of an ANSYS Academic Research License to Instituto de Óptica (CSIC).

\section{References}

1. Schanzlin DJ, Asbell PA, Buris TE, Durrie DS. The intrastromal corneal ring segments. Phase II results for the correction of myopia. Ophthalmology. 1997;104:1067-1078.

2. Cochener B, Savary-LeFloch G, Colin J. Effect of intrastromal corneal ring segment shift on clinical outcome: one year results for low myopia. J Cataract Refract Surg. 2000;26:978986.

3. Ruckhofer J, Stoiber J, Twa MD, Grabner G. Correction of astigmatism with short arc-length intrastromal corneal ring segments: preliminary results. Ophthalmology. 2003;110:516524 .

4. Blavatskaia, DED. The use of intralamellar homoplasty in order to reduce refraction of the eye. Überstzt Aus Oftalmol $\mathrm{Zh}$. 1966; 7:530-537.

5. Flemming JF, Wan WL, Schanzlin DJ. The theory of corneal curvature change with the intrastromal corneal ring. CLAOJ. 1989;15:146-150.

6. Siepser SB, inventor; Kera Associates, assignee. Device used to change corneal curvature. US patent 4,976,719. December 11, 1990.

7. Simon G, Parel JM, Lee W, Kervick GN. Gel injection adjustable keratoplasty. Graefes Arch Clin Exp Ophthalmol. 1991;229: 418-424.

8. Siganos D, Ferrara P, Chatzinikolas K, Bessis N, Papastergiou G. Ferrara intrastromal corneal rings for the correction of keratoconus. J Cataract Refract Surg. 2002;28:1947-1951.

9. Kubaloglu A, Cinar Y, Sari ES, Koytak A, Ozdemir B, Ozertürk Y. Comparison of 2 intrastromal corneal ring segment models in the management of keratoconus. J Cataract Refract Surg. 2010;36:978-985.

10. Siganos CS, Kymionis GD, Kartakis N, Theodorakis MA, Astyrakakis N, Pallikaris IG. Management of keratoconus with Intacs. Am J Ophthalmol. 2003;135:64-70.

11. Ortiz S, Pérez-Merino P, Alejandre N, Gambra E, Jimenez-Alfaro I, Marcos S. Quantitative OCT-based corneal topography in keratoconus with intracorneal ring segments. Biomed Opt Express. 2012;3:814-824.

12. Djotyan GP, Kurtz RM, Fernández DC, Juhasz T. An analytically solvable model for biomechanical response of the cornea to refractive surgery. J Biomech Eng. 2001;123:440-445.

13. Patel S, Marshall J, Fritzke FW III. Model for deriving the optical performance of the myopic eye corrected with an intracorneal ring. J Refract Surg. 1995;11:248-252.

14. Silvestrini TA, Mathis ML, Loomas BE, Burris TE. A geometric model to predict the change in corneal curvature from the intrastromal corneal ring (ICR). Invest Ophthalmol Vis Sci. 1994;35(suppl):2023.

15. Piñero DP, Alio JL, Teus MA, Barraquer RI, Uceda-Montañés A. Modeling the intracorneal ring segment effect in keratoconus using refractive, keratometric, and corneal aberrometric data. Invest Ophthalmol Vis Sci. 2010;51:5583-5591.

16. Pinsky PM, Datye DV. A microstructurally-based finite element model of the incised human cornea. J Biomech. 1991;24:907922.

17. Glass DH, Roberts CJ, Litsky AS, Weber PA. A viscoelastic biomechanical model of the cornea describing the effect of viscosity and elasticity on hysteresis. Invest Ophthalmol Vis Sci. 2008;49:3919-3926. 
18. Pandolfi A, Manganiello F. A model for the human cornea: constitutive formulation and numerical analysis. Biomech Model Mechanobiol. 2006;5:237-246.

19. Anderson K, El-Sheikh A, Newson T. Application of structural analysis to the mechanical behaviour of the cornea. $J R S o c$ Interface. 2004;1:3-15.

20. Roy AS, Dupps WJ Jr. Patient-specific computational modeling of keratoconus progression and differential response to collagen cross-linking. Invest Ophthalmol Vis Sci. 2011;52: 9174-9187.

21. Nakayasu K, Tanaka M, Konomi H, Hayashi T. Distribution of types I, II, III, IV and V collagen in normal and keratoconus corneas. Ophthalmic Res. 1986;18:1-10.

22. Daxer A, Fratzl P. Collagen fibril orientation in the human corneal stroma and its implications in keratoconus. Invest Ophthalmol Vis Sci. 1997;38:121-129.

23. Meek KM, Tuft SJ, Huang Y, et al. Changes in collagen orientation and distribution in keratoconus corneas. Invest Ophthalmol Vis Sci. 2005;46:1948-1956.

24. Oxlund H, Simonsen AH. Biochemical studies of normal and keratoconus corneas. Acta Ophthalmol (Copenh). 1985;63: 666-669.

25. Edmund C. Corneal elasticity and ocular rigidity in normal and keratoconic eyes. Acta Ophthalmol (Copenh). 1988;66:130140.

26. Maeda N, Fujikado T, Kuroda T, et al. Wavefront aberrations measured with Hartmann-Shack sensor in patients with keratoconus. Ophthalmology. 2002;109:1996-2003.

27. Barbero S, Marcos S, Merayo-Lloves J, Moreno-Barriuso E. Validation of the estimation of corneal aberrations from videokeratography in keratoconus. J Refract Surg. 2002;18: 263-270.

28. Rabinowitz YS. Keratoconus. Surv Ophthalmol. 1998;42:297319.

29. Wilson SE, Lin DT, Klyce SD. Corneal topography of keratoconus. Cornea. 1991;10:2-8.

30. Hennighausen H, Feldman ST, Bille JF, McCulloch AD. Anteriorposterior strain variation in normally hydrated and swollen rabbit cornea. Invest Ophthalmol Vis Sci. 1998;39:253-262.

31. Bryant MR, McDonnell PJ. Constitutive laws for biomechanical modeling of refractive surgery. J Biomech Eng. 1996;118:473481 .

32. Kampmeier J, Radt B, Birngruber R, Brinkmann R. Thermal and biomechanical parameters of porcine cornea. Cornea. 2000;19:355-363.

33. Girard MJA, Suh J-KF, Bottlang M, Burgoyne CF, Downs JC. Biomechanical changes in the sclera of monkey eyes exposed to chronic IOP elevations. Invest Ophthalmol Vis Sci. 2011;52: 5656-5669.

34. Olsen TW, Sanderson S, Feng X, Hubbard WC. Porcine sclera: thickness and surface area. Invest Ophthalmol Vis Sci. 2002; 43:2529-2532.

35. Coskunseven E, Kymionis GD, Jankov MR. Complications of intra corneal ring segment implantation with femtosecond laser channel creation in patients with keratoconus (explanations and solutions). J Emmetropia. 2010;1:221-228.

36. Stefancu A-I, Melenciuc S-C, Budescu M. Finite element analysis of frictional contacts. Bul Inst Politeh Iaşi. 2011:58: 131-139.
37. Piñero DP, Alió JL, El Kady B, Pascual I. Corneal aberrometric and refractive performance of 2 intrastromal corneal ring segment models in early and moderate ectatic disease. $J$ Cataract Refract Surg. 2010;36:102-109.

38. Miranda D, Sartori M, Francesconi C, Allemann N, Ferrara P, Campos M. Ferrara intrastromal corneal ring segments for severe keratoconus. J Refractive Surg. 2003;19:645-653.

39. Brooks AM, Robertson IF, Mahoney A-M. Ocular rigidity and intraocular pressure in keratoconus. Aust J Ophthalmol. 1984; $12: 317-324$

40. Ferrara G, Torquetti L, Ferrara P, Merayo-Lloves J. Intrastromal corneal ring segments: visual outcomes from a large case series. Clin Exp Ophthalmol. 2012;40:433-439.

41. Pérez-Merino P, Parra F, Ibares-Frías L, et al. Clinical and pathological effects of different acrylic intracorneal ring segments in corneal additive surgery. 2012;6:2572-2579.

42. Gefen A, Shalom R, Elad D, Mandel Y. Biomechanical analysis of the keratoconic cornea.J Mech Behav Biomed Mater. 2009; 2:224-236.

43. Nash IS, Greene PR, Foster CS. Comparison of mechanical properties of keratoconus and normal corneas. Exp Eye Res. 1982;35:413-424.

44. Andreassen TT, Simonsen AH, Oxlund H. Biomechanical properties of keratoconus and normal corneas. Exp Eye Res. 1980;31:435-441.

45. Twa MD, Ruckhofer J, Kash RL, Costello M, Schanzlin DJ. Histologic evaluation of corneal stroma in rabbits after intrastromal corneal ring implantation. Cornea. 2003;22: 146-152.

46. Wollensak G, Spoerl E, Seiler T. Stress-strain measurements of human and porcine corneas after riboflavin-ultraviolet-Ainduced cross-linking. J Cataract Refract Surg. 2003;29: 1780-1785.

47. Litwiller DV, Lee SJ, Kolipaka A, et al. MR elastography of the ex vivo bovine globe. J Magn Reson Imaging. 2010;32:44-51.

48. Wollensak G, Iomdina E. Biomechanical and histological changes after corneal crosslinking with and without epithelial debridement. J Cataract Refract Surg. 2009;3:540-546.

49. Kling S, Remon L, Pérez-Escudero A, Merayo-Lloves J, Marcos S. Corneal biomechanical changes after collagen cross-linking from porcine eye inflation experiments. Invest Ophthalmol Vis Sci. 2010;51:3961-3968.

50. Scarcelli G, Yun SH. Confocal Brillouin microscopy for threedimensional mechanical imaging. Nat Photon. 2008;2:39-43.

51. Dorronsoro C, Pascual D, Pérez-Merino P, Kling S, Marcos S. Dynamic OCT measurement of corneal deformation by an air puff in normal and cross-linked corneas. Biomed Opt Express. 2012;3:473-487.

52. Morishige N, Wahlert AJ, Kenney MC, et al. Second-harmonic imaging microscopy of normal human and keratoconus cornea. Invest Ophthalmol Vis Sci. 2007;48:1087-1094.

53. Bueno JM, Gualda EJ, Giakoumaki A, Péres-Merino P, Marcos S, Artal P. Multiphoton microscopy of ex vivo corneas after collagen cross-linking. Invest Ophthalmol Vis Sci. 2011;52: 5325-5331.

54. Ortiz S, Siedlecki D, Remon L, Marcos S. Optical coherence tomography for quantitative surface topography. Appl Optics. 2009;48:6708-6715. 